\title{
EL IMPACTO CULTURAL Y SOCIAL DE LOS EVENTOS CELEBRADOS EN DESTINOS TURÍSTICOS. LA PERCEPCIÓN DESDE EL PUNTO DE VISTA DE LOS ORGANIZADORES
}

\author{
Francesc Gonzàlez Reverté \\ Soledad Morales Pérez. \\ Universitat Oberta de Catalunya
}

\section{RESUMEN}

El objetivo de este estudio es analizar la existencia de asociación entre los impactos socioculturales generados por los eventos celebrados en localidades turísticas de Cataluña y la vocación turística de los mismos. Para ello los eventos analizados son clasificados en dos tipologías en función de si plantean objetivos turísticos en su misión y de si su programación contiene elementos adaptados a los gustos de los turistas. Los datos necesarios fueron recogidos por medio de una encuesta en línea realizada a organizadores de eventos y se basa en una muestra de 263 casos. La metodología establecida consistió en cruzar la opinión de los organizadores (impactos socioculturales percibidos de la celebración de eventos) con los diferentes tipos de eventos según su vocación turística mediante tablas de contingencia y la aplicación de un test de significación. El principal resultado obtenido es la confirmación de la existencia de una relación significativa entre el impacto sociocultural y el nivel de turistificación de los eventos. En concreto, estos eventos, aportan impactos positivos sobre el sentido del lugar, la identidad y el bienestar de la comunidad donde se celebran. Los efectos positivos sobre la identidad local obtienen valores especialmente altos. En nuestra opinión, esto está relacionado con la forma en que los actores sociales de los destinos turísticos (en nuestro caso los organizadores de eventos) tienden a construir la identidad incluyendo las actividades turísticas como elemento intrínseco de su manera de ser. Los eventos turísticos, que muy a menudo basan sus narraciones sobre

Recibido: 21 de diciembre de 2015

Devuelto para su revisión: 4 de mayo de 2016

Aceptado: 25 de julio de 2016

Estudios de Economía y Empresa. Universitat Oberta de Catalunya. Av. Tibidabo, 39. 08035 BARCELONA (España).E-mail: fgonzalezre@uoc.edu, smoralespe@uoc.edu 
la identidad y la historia local, se integran, por lo tanto, fácilmente en el imaginario local y son percibidos como activos con capacidad para proyectar el desarrollo social y cultural local.

Palabras clave: Eventos, impactos sociales y culturales, identidad, comunidad local, destinos turísticos, Organizadores de eventos.

\title{
Cultural and social impacts of events held in tourism destinations. A managers' perception point of view
}

\begin{abstract}
The aim of this study is to analyze the relationship between the touristification of events taking place at mature tourist destinations and the sociocultural impact they cause in the local community. To this aim these events were classified according to their degree of involvement in tourism and event managers were surveyed about their potential impact. Data was gathered by means of a survey carried out with event organizers and is based on a sample of 263 cases. We measured the correlation between different items of social and cultural impact on the local community and five types of events classified according to presence or absence of tourism-oriented goals. The main result obtained is the confirmation of a significant relationship between sociocultural impact and the touristification level of events. The celebration of tourist festivals and events in Catalan tourist destinations has a positive impact on sense of place, place identity and community well-being. Positive effects on local identity obtain particularly high values. In our view, this is related to the way social actors in tourist destinations build the place identity by including tourist events as part of their image, given that these events often base their narratives on local identity and history.
\end{abstract}

Keywords: Events, social and cultural impacts, identity, local community, tourist destinations, Event managers.

\section{INTRODUCCIÓN. LA CELEBRACIÓN DE EVENTOS EN DESTINOS TURÍSTICOS}

Uno de los signos más claros de la consolidación de la experiencia turística ha sido el creciente aumento del número de eventos culturales y turísticos celebrados en los últimos años. El crecimiento de estos eventos en las áreas rurales, ciudades y destinos turísticos ha sido constante. Una amplia variedad de estrategias los ha impulsado y, aunque estas pueden variar de acuerdo a cada caso, en general están relacionadas con el aprovechamiento de la capacidad que los eventos tienen para generar desarrollo local y atraer a los turistas (Faulkner et al, 2000; Giampiccolo, Shawn \& Nauright, 2013; Small et al, 2005; Stokes \& Jago, 2007). La capacidad de los eventos para generar experiencias únicas e irrepetibles (no hay dos celebraciones idénticas de un evento) (Getz, 2007), el coste relativamente bajo de ponerlos en marcha (comparado con el coste de otras medidas de atracción de turistas basadas en la construcción de una infraestructura física como, por ejemplo, un centro de interpretación), su potencial para crear un ambiente urbano y un sentido de lugar y sus 
efectos visibles a corto plazo (la atracción de visitantes, el impulso de la actividad en los espacios públicos, la mejora de la imagen urbana, el aumento de la visibilidad, etc.) (Pugh \& Wood, 2004, Ritchie, Sanders, \& Mulas, 2007; Shibli, \& Coleman, 2005) son algunas de las razones que explican su frecuente utilización como estrategia de revitalización urbana y turística (González \& Morales, 2009; Higham \& Ritchie, 2011; Richards \& Palmer, 2010).

Desde el punto de vista de los destinos turísticos un aspecto fundamental que explica la capacidad de seducción de los eventos es su potencial para reforzar la identidad local, aspecto que permite individualizar y diferenciar al destino respecto de los competidores (Carlsen, Robertson \& Ali-Knight, Jane, 2008; Derret, 2003; De Bres \& Davis, 2001; Small, 2008). Es evidente que esta posibilidad está en armonía con la posibilidad de crear productos basados en la especificidad de los lugares y la autenticidad, estrategias que algunos destinos promueven como una alternativa al modelo tradicional del turismo. Un segundo aspecto a destacar es que los eventos tienen efectos sobre el desarrollo social de las comunidades que los organizan (Deery \& Jago, 2010, Fredline, Jago \& Deery, 2003, Reid, 2008). Además, otro resultado de la celebración de eventos es el aumento de la participación pública, con lo que contribuyen a la construcción de la cohesión social en la comunidad (González \& Morales, 2009, Mackellar, 2006; González \& Miralbell, 2009; Moscardo, 2008).

No obstante, el uso de eventos en ciudades y localidades turísticas no solamente aporta beneficios económicos y sociales sino que también se asocia frecuentemente con la presencia de impactos sociales y culturales negativos sobre la comunidad local: desplazamiento de los residentes (Eisinger, 2000; Misener \& Mason, 2006b), homogeneización cultural (Richards \& Wilson, 2006; Mc Cartney \& Osti, 2007) y desnaturalización de las narrativas y de la identidad local para adaptarlas al evento (Ronstrom, 2011). Para comprender plenamente el impacto potencial de los eventos en los destinos turísticos hay una necesidad de profundizar en estos temas.

Nuestra investigación pretende contribuir al estudio de la relación entre la celebración de eventos en localidades turísticas en Cataluña y la percepción de su impacto social y cultural. La investigación se basa en el diseño de una encuesta en línea dirigida a los responsables de la organización de eventos públicos y privados en los destinos turísticos de Cataluña con el objetivo de medir su percepción acerca del impacto social y cultural derivado de la celebración de estos eventos. Los eventos se han clasificado en cinco tipos diferentes a partir de dos categorías: la orientación turística del evento y su grado de turistificación (véase la tabla 1). Para determinar la orientación turística de un evento se ha tenido en cuenta como variable los objetivos que los organizadores persiguen con la celebración del evento, así como cuál es el objetivo principal de entre los que se mencionan. La segunda categoría toma como variables la existencia de elementos especialmente diseñados para los turistas entre las actividades que constan en el programa del evento y el número de turistas que han asistido al evento durante las tres últimas ediciones. La primera categoría permite diferenciar entre dos tipos de eventos: 1) eventos que declaran tener como objetivo principal la promoción del turismo o la atracción de turistas (T1 o eventos orientados al turismo) y 2) eventos en que la promoción del turismo se incluye en sus objetivos aunque no se considera el principal (T2 o eventos parcialmente orientados al turismo).

La segunda categoría permite distinguir tres tipos de eventos: 1) eventos que han experimentado un aumento significativo de los turistas extranjeros en las últimas edicio- 
nes (T3 o eventos con capacidad de atracción turística); 2) eventos que adaptan parte de su programa al gusto de los mercados globales (T4 o eventos transformados por el turismo) y 3) eventos que han sido especialmente diseñados para los turistas y visitantes (T5 o eventos turísticos). Por último, las respuestas sobre el impacto social y cultural del evento, obtenidas a partir de diferentes variables, se analizan de forma agregada para cada uno de los cinco tipos de eventos identificados. Con ello se pretende analizar el grado de relación existente entre la orientación turística de un evento y el grado de turistificación del mismo y los impactos sociales y culturales que genera su celebración.

Tabla 1

CLASIFICACIÓN DE LOS EVENTOS POR TIPOS

\begin{tabular}{|l|l|}
\hline Tipo de evento & Ejemplos \\
\hline T1. Eventos orientados al turismo & Ferias, mercados, jornadas gastronómicas \\
\hline $\begin{array}{l}\text { T2. Eventos parcialmente orientados al } \\
\text { turismo }\end{array}$ & $\begin{array}{l}\text { Eventos de promoción de productos } \\
\text { locales, fiestas tradicionales, festivales }\end{array}$ \\
\hline $\begin{array}{l}\text { T3. Eventos con capacidad de atracción de } \\
\text { turistas }\end{array}$ & $\begin{array}{l}\text { Festivales de prestigio, grandes ferias, } \\
\text { celebraciones tradicionales }\end{array}$ \\
\hline T4. Eventos transformados por el turismo & $\begin{array}{l}\text { Ferias, festivales, eventos de promoción de } \\
\text { productos turísticos locales }\end{array}$ \\
\hline T5. Eventos turísticos & $\begin{array}{l}\text { Festivales, mercados medievales, jornadas } \\
\text { gastronómicas, eventos temáticos }\end{array}$ \\
\hline
\end{tabular}

Fuente: elaboración propia.

\section{MARCO CONCEPTUAL. EL IMPACTO SOCIOCULTURAL DE LA CELE- BRACIÓN DE EVENTOS}

Cada vez hay más conciencia entre los investigadores de la necesidad de medir el impacto sociocultural de festivales y eventos para evitar que la insatisfacción de la comunidad local amenace su éxito a largo plazo (Samll, Edwards \& Sheridan, 2005; Mair \& Whitford, 2013). Según Deery \& Jago gran parte de la investigación sobre los impactos sociales que los eventos producen en las comunidades locales se ha centrado en el desarrollo de escalas para evaluar sus beneficios y costes sociales, determinar cómo la percepción de los residentes afectan el apoyo local hacia los eventos, así como en recomendar medidas a las autoridades locales para mejorar su impacto social (Deery \& Jago, 2010).

Varios autores sugieren que el impacto socio-cultural es el resultado de la interacción entre los turistas, el destino turístico y los residentes locales. Festivales y eventos afectan a destinos en su conjunto por lo que siempre hay un considerable impacto sociocultural potencial. Otros autores, de acuerdo con la teoría del intercambio social, afirman que existe una relación directa entre la percepción del impacto del turismo por parte de los actores sociales y factores como su relación personal con este sector (Pizam, 1978), la proximidad a la actividad turística (Sheldon \& Var, 1984; Keogh 1990) y el grado de contacto directo con los turistas (Pizam, Milman \& King, 1994; Rothman). 
Nuestro estudio tiene la intención de aportar un punto de vista diferente a la investigación sobre los impactos de eventos partiendo de la percepción de los propios organizadores. El interés de este estudio radica en el hecho de que se analiza no solamente los efectos que el turismo tiene sobre la celebración de eventos locales diseñados para residentes, sino que también se tiene en cuenta los eventos pensados para los turistas. Esto exige superar la dificultad que supone el hecho que los eventos celebrados en localidades turísticas con frecuencia asumen formatos híbridos que van dirigidos tanto a los residentes locales como a los visitantes (tal es el caso de los eventos dirigidos a la promoción de productos locales o los festivales de verano). Para solventarlo se han clasificado los eventos a partir de la finalidad que los propios organizadores otorgan al evento, especialmente si incluyen el turismo o no lo incluyen entre sus objetivos. Las diferentes variables para caracterizar el impacto social y cultural de los eventos han sido seleccionadas a partir de una revisión bibliográfica sobre el tema. Sin embargo, tenemos que tener en cuenta que existen múltiples enfoques sobre los impactos de la celebración de eventos, aspecto que impide un enfoque holístico. Poseer un conocimiento directo de la zona de estudio fue un factor determinante a la hora de hacer la selección final de las variables para analizar el impacto.

En concreto, hemos tenido en cuenta cuatro aspectos relacionados con el fortalecimiento del sentido de comunidad (Sharpley \& Stone, 2012). En primer lugar se analizan los efectos de los eventos sobre la identidad cultural local y regional (De Bres \& Davis, 2001; Ekman, 1999; Gonzàlez et al., 2012; Waterman, 1998). Un segundo tipo de impacto se refiere a la capacidad de los eventos para fortalecer el sentido del lugar (Avery, 2000; Derrett, 2003). Un tercer tipo de impacto considerado es la capacidad de los eventos para fortalecer el sentido de comunidad y el capital social (Derrett, 2003, Winkle \& Woosnam, 2014). Por último, se analizan los impactos vinculados al fortalecimiento de la sensación de bienestar entre la comunidad local cuando se celebran eventos. (Jepson \& Clarke, 2013).

Muchos de los eventos incluidos en el estudio son festivales organizados desde y para la comunidad local apoyados en el patrimonio histórico local, lo que permite el fortalecimiento de la cultura y la historia local y por lo tanto mejorar el sentido de identidad de los asistentes (De Bres \& Davis, 2001). En muchas ocasiones el evento actúa como escaparate de los valores sociales y culturales que la comunidad local pretende mostrar, aspecto que aumenta la consolidación de la identidad local. Sin embargo, es importante tener en cuenta que precisamente los turistas se interesan por los eventos con un alto contenido de autenticidad. Esto hace que aumente el riesgo de vincular el evento a estrategias de desarrollo turístico y a dejar de lado los aspectos sociales y culturales de la celebración (Getz, 1989; Mehmetoglu \& Ellingson, 2005). En añadidura, los problemas con los residentes pueden surgir cuando un evento se altera para satisfacer mejor las necesidades de los turistas (Mules, 2004; Xiao \& Smith, 2004). Los posibles efectos negativos generados cuando los acontecimientos son vistos como una exhibición de poder y hegemonía política de un grupo social hegemónico o de la comunidad sobre las minorías o menos poderosos grupos étnicos es otro tipo de riesgo a tener en cuenta (Jeong \& Santos, 2004).

Celebrar eventos permite también aumentar el sentido del lugar entre la comunidad local, ya que mejoran la imagen y el posicionamiento de los destinos. Los festivales se utilizan a menudo en los destinos turísticos para posicionar la marca turística y para fortalecer su imagen, aprovechando su capacidad para diferenciar y singularizar los lugares 
que los albergan (Richards \& Wilson, 2004). En este contexto, el objetivo es sacar provecho de una imagen mejorada y obtener nuevas inversiones, nuevos negocios y aumentar el número de turistas con el fin de dinamizar los sectores productivos locales (Sharpley \& Stone, 2012) y regenerar el tejido urbano (Smith, 2012). La mejora de la identidad del lugar a través de la celebración de eventos en los destinos turísticos requiere que los eventos se adapten al gusto de los turistas y satisfagan sus expectativas como espectáculo. Esto puede ser interpretado por los asistentes como una pérdida de la autenticidad del acto (MacLeod, 2006), aunque también como un proceso de reformulación de la cultura local (Wood, 1998). Por su parte, las relaciones entre la celebración de eventos y el capital social han sido objeto de diferentes estudios (Wilks, 2012; Misener, 2013). El capital social puede ser un factor importante para el desarrollo local y regional si se combina con otros factores (Moscardo, 2008). Además también actúa como un agente de cohesión social para mantener las actividades que refuerzan la vida en común de una comunidad y para aumentar la capacidad de gestionar los recursos turísticos de una forma más sostenible. Tres aspectos básicos de los vínculos que existen entre el capital social y los eventos son considerados en nuestra investigación: el consenso social que generan en la comunidad (Arcodia \& Whitford, 2006; Gunsoy \& Hannam, 2013), la capacidad para organizar redes internas y externas, así como de generar las relaciones sociales de reciprocidad (Misener \& Mason, 2006a) y la confianza existente con el entorno institucional (González et al., 2011). El capital social se entiende, en este trabajo, como un recurso que aporta elementos intangibles que fortalecen una comunidad. Por tanto, podemos suponer que si la celebración de eventos refuerza los mecanismos del capital social local, el sentido de comunidad también puede verse afectada de manera positiva.

Nahapiet y Ghoshal definen el capital social como "la suma de los recursos reales y potenciales incrustados en, disponibles a través de, y derivados de la red de relaciones que posee una unidad individual o social" (Nahapiet \& Ghoshal, 1998: 243). Es decir, el capital social es visto como un recurso básico que las organizaciones utilizan para crear valor y capital intelectual, así como los bienes de producción, y un elemento que aumenta la eficiencia de las organizaciones mediante la reducción de sus costes de transacción. El capital social es un término amplio y el concepto se ha teorizado desde diferentes enfoques que van desde una visión a escala macro que pone de relieve el funcionamiento de las redes sociales y su potencial para el bien público indirecto hasta un enfoque a nivel micro que pone el énfasis en destacar las acciones personales y beneficios potenciales para los individuos (Morales, 2013; Wilks, 2012). Según Coleman, el capital social es un conjunto de recursos socio-estructurales que constituyen un activo para las personas y facilitan ciertas acciones comunes entre los miembros que incluye esta estructura (Coleman, 1988). Bourdieu, por su parte, afirma que el capital social es la suma de los recursos vinculados a una red social extensa y de larga duración con relaciones más o menos institucionalizadas y que es reconocida por los miembros de una comunidad. Por lo tanto, el capital social de cualquier actor en particular dependerá del alcance que la red pueda tener y su capacidad para ser movilizada de manera eficiente, así como del volumen de capital que cada actor de la red pueda poseer (Bourdieu, 1983).

La visión de capital social que ofrece Putnam encaja perfectamente con el objeto de este estudio, que tiene como objetivo medir el impacto colectivo que los eventos causan 
en las comunidades turísticas. Según Putnam, el capital social incluye elementos de organización social, tales como redes, normas y confianza que proporcionan la coordinación y la cooperación necesaria para obtener un beneficio común (Putnam, 1993; 2000). Se trata de un conjunto de relaciones, normas de comportamiento, valores, obligaciones y canales de información que actúan como vehículo para la obtención de los recursos y beneficios en las organizaciones y comunidades con lazos de asociación y compromiso cívico establecidos y redes sociales desarrolladas (Woolcock, 1998; Cooke \& Wills, 1999; Woolcock \& Narayan, 2000; Knorringa \& van Staveren, 2007). El capital social actúa como un factor que construye el compromiso cívico y es un indicador de la salud de la comunidad que transforma los recursos de los individuos en acciones colectivas. A pesar del hecho de que el capital es un término con una definición compleja y polisémica, existe un acuerdo general sobre el hecho de que se refiere a la capacidad de los individuos para obtener beneficios sobre la base de su pertenencia a estructuras sociales (Harpham, Grant, \& Thomas, 2002).

Finalmente, se considera que los eventos pueden contribuir de diferentes maneras a la aparición de impactos sociales de larga duración en la comunidad local si son capaces de mejorar la sensación de bienestar de dicha comunidad. La capacidad de los eventos para impulsar desarrollo social permite, a su vez, mejorar las relaciones entre los miembros de una comunidad, motivar a los individuos a que participen más en los asuntos locales, aumentar el orgullo cívico local y mejorar la percepción en términos de calidad de vida. Algunos de los aspectos positivos que los eventos pueden aportar en materia de bienestar social son la mejora del acceso a la cultura, la inclusión social de los grupos marginados y el fortalecimiento del trabajo voluntario. Sin embargo, también deben tenerse en cuenta algunos aspectos negativos. La teoría del intercambio social sugiere que ciertos grupos sociales no obtienen beneficios de la celebración de eventos, pero sí que sufren algunos costes y aspectos negativos de estos eventos, por lo que puede derivar en casos de insatisfacción, desventaja social e incluso conducir a la acción política o de protesta (Fredline \& Faulkner, 1998).

\section{METODOLOGÍA}

Los datos utilizados para el estudio se reunieron mediante una encuesta en línea llevada a cabo entre diciembre de 2007 y enero de 2008 con el objetivo de determinar la relación entre el desarrollo local y la realización de eventos culturales. Las encuestas fueron enviadas directamente a los organizadores a través de las direcciones de correo electrónico registradas en el censo de eventos culturales en Cataluña. Cuando esta información no estaba disponible o era incorrecta, se hicieron llamadas telefónicas para encontrar a una persona de contacto y enviar el cuestionario vía correo electrónico. Una semana antes de enviar las encuestas a todos los organizadores se envió un correo electrónico donde se les informaba de la naturaleza del estudio que se estaba llevando a cabo; y se añadió una carta oficial de la institución que había solicitado el estudio. Para asegurar que los municipios incluidos en el estudio responden a un perfil turístico se excluyeron de la muestra las localidades catalanas con menos de 3.000 camas turísticas reguladas o menos de 150 plazas de turismo rual. El resultado final incluye localidades que responden a un perfil variado de destinos turísticos (de sol y playa, ciudades históricas, estaciones de montaña y centros turísticos rurales). 
El perfil de los entrevistados corresponde a personas que, en el momento de iniciar la investigación, estaban a cargo de la gestión de eventos (presidentes de asociaciones sociales o culturales, promotores privados, técnicos en turismo o dinamización económica y cargos de la administración local, gestores culturales, etc.), incluyendo a los gerentes y a los directores artísticos. A pesar del sesgo potencial generado por el hecho de que las respuestas sobre los impactos se fundamentan en el punto de vista de los organizadores, hay una serie de razones y ventajas prácticas que hacen que sus opiniones sean valiosas para el estudio. El uso de una encuesta a los organizadores a partir de una muestra ha permitido analizar un número importante de los eventos celebrados en Cataluña por lo que se ha partido de una visión representativa y se ha evitado realizar generalizaciones a partir de uno o diversos casos de estudio. Los organizadores eran los referentes ideales puesto que son los actores que disponen de mayor información de primera mano sobre la gestión y el funcionamiento de estos eventos. Además con frecuencia son los promotores directos de los acontecimientos y por lo tanto conocen perfectamente su historia y antecedentes. Finalmente, los organizadores de eventos son actores sociales muy importantes en la creación y gestión de las actividades del destino turístico y por lo tanto resultan actores representativos como grupo de opinión local.

La encuesta se estructuró en diferentes bloques. Uno de los bloques se refería a la relación entre los acontecimientos y la creación de capital social. Un segundo bloque preguntaba acerca de los impactos socio-culturales percibidos a nivel local como consecuencia de la celebración de eventos. En un tercer bloque se preguntaban diversos aspectos vinculados con las relaciones institucionales promovidas mediante la celebración del evento. La encuesta se envió a 2.045 eventos registrados en el censo del Departamento de Cultura de la Generalitat de Catalunya, obteniéndose 263 respuestas válidas (12\% del total de eventos). Se enviaron cuestionarios a diferentes tipos de fiestas y eventos (festivales de arte, festivales de música tradicional, mercados medievales, eventos deportivos, ferias y mercados, etc.) celebradas en Cataluña a partir de un muestreo aleatorio estratificado por comarcas. Los resultados de la encuesta tienen un margen de error de $+/-3 \%$, con un intervalo de confianza del 95\% $(\mathrm{p}=\mathrm{q}=50 \%)$.

Los impactos sociales y culturales relacionados con la celebración de eventos se midieron a partir de 26 variables extraídas de los datos proporcionados por las encuestas (véase la Tabla 2). Un primer grupo de variables ofrece información sobre el impacto de la celebración de eventos sobre la identidad cultural local. Se refieren específicamente a la participación de artistas locales en el programa del evento, a los valores de identidad reflejados en el evento (incluyendo el uso de la lengua catalana) y a la autenticidad que transmite el evento (representación histórica fidedigna, presencia de relatos tradicionales o culturales sobre los que se basa el evento, presencia de valores asociados a la cultura local, etc.). Un segundo grupo de variables pretende evaluar los impactos sobre el sentido del lugar. Estas variables reflejan el grado en que un evento actúa como fuente de orgullo local, el grado de visibilidad externa y los efectos en la imagen turística y en la marca de un destino. El tercer grupo de variables evalúa el impacto de los acontecimientos sobre el sentido de comunidad. Para ello se utilizan como variables dos elementos (la confianza y la participación social) que fortalecen el capital social entre los miembros de una comunidad. El cuarto grupo de variables incluye aquellos aspectos relacionados con 


\section{Tabla 2}

\section{VALORES MEDIOS DE LAS VARIABLES UTILIZADAS PARA EL CÁLCULO DE LOS IMPACTOS SOCIALES Y CULTURALES DERIVADOS DE LA CELEBRACIÓN DE EVENTOS}

\begin{tabular}{|c|c|c|}
\hline Indicador & Media & DT \\
\hline $\begin{array}{l}\text { Los organizadores del evento mantienen una relación de plena confianza y reciprocidad } \\
\text { con la administración pública }\end{array}$ & 4,26 & 0,303 \\
\hline $\begin{array}{l}\text { El evento permite aumentar las posibilidades de ocio y de entretenimiento entre la } \\
\text { población local }\end{array}$ & 4,17 & 0,775 \\
\hline El evento genera impactos sociales y culturales positivos para la población local & 3,95 & 0,903 \\
\hline La participación de los residentes en el evento es considerable & 3,94 & 0,904 \\
\hline El evento es una fuente de orgullo para los residentes & 3,93 & 0,97 \\
\hline El evento favorece la socialización de los residentes & 3,87 & 0,88 \\
\hline Los residentes forman parte de la gestión y organización del evento & 3,79 & 0,401 \\
\hline Las asociaciones y entidades locales participan en el evento & 3,75 & 1,005 \\
\hline La presencia de voluntarios es esencial para la celebración del evento & 3,66 & 1,448 \\
\hline El evento reproduce los valores de la identidad local & 3,66 & 0,997 \\
\hline El evento reproduce los valores de la identidad catalana & 3,56 & 1,005 \\
\hline El evento muestra el pasado y la historia local de forma fidedigna & 3,56 & 1,001 \\
\hline El evento proporciona una interpretación adecuada de la historia local para los residentes & 3,56 & 1,001 \\
\hline $\begin{array}{l}\text { Los recursos destinados a la financiación del evento deberían dedicarse a cubrir } \\
\text { necesidades más urgentes de la localidad }\end{array}$ & 3,53 & 1,094 \\
\hline El evento muestra de forma adecuada la manera tradicional de vida local & 3,5 & 0,989 \\
\hline El evento muestra los valores culturales y tradicionales locales & 3,5 & 0,955 \\
\hline La programación del evento cuenta con una profusa representación de artistas catalanes & 3,35 & 1,19 \\
\hline El evento ofrece nuevas actividades culturales para la población local & 3,33 & 1,151 \\
\hline El evento fortalece la cultura catalana desde el punto de vista de la lengua & 3,29 & 1,083 \\
\hline El evento refuerza la imagen de marca turística local & 3,26 & 1,201 \\
\hline El evento permite la mejora de los espacios públicos urbanos & 3,08 & 1,144 \\
\hline El evento despierta la atención de los medios de comunicación internacionales & 2,25 & 1,055 \\
\hline $\begin{array}{l}\text { Las molestias físicas generadas por el evento (masificación, ruido, residuos, etc.) } \\
\text { provocan tensiones con los residentes locales }\end{array}$ & 1,92 & 0,736 \\
\hline La organización y celebración del evento provoca tensiones con la administración local & 1,84 & 0,735 \\
\hline $\begin{array}{l}\text { La presencia de turistas asistentes al evento es percibida como una molestia por parte de } \\
\text { los residentes locales }\end{array}$ & 1,02 & 0,891 \\
\hline
\end{tabular}

Fuente: elaboración propia.

la mejora de la sensación de bienestar entre la comunidad local e incluye variables como el estímulo de la socialización y el entretenimiento, la creación de actividades culturales paralelas, el uso del espacio público y la generación de beneficio social generados por el evento. Finalmente, se ha tenido en cuenta una serie de variables para valorar algunos de los impactos negativos derivados de la celebración de eventos. Estas variables incluyen el impacto por la presencia de turistas, los impactos físicos que perturban a la población 
local, la existencia de controversias entre los organizadores y el gobierno local y los costes económicos de la celebración de eventos. Las respuestas obtenidas se midieron a partir de una escala Likert de 5 ítems, donde 1 significa "no estoy nada de acuerdo con la afirmación" y 5 significa "estoy muy de acuerdo con la afirmación". Para establecer la significación entre la asociación de las diferentes variables de impacto y los diversos tipos de eventos se utilizó la prueba de ji-cuadrado (con 4 grados de libertad), tomándose en cuenta los valores de significación inferiores a 0,005 .

\section{RESULTADOS}

El análisis de los resultados muestra, en primer lugar que los eventos con carácter turístico celebrados en los destinos catalanes generan impactos sociales y culturales positivos. Los eventos orientados al turismo (T1) tienen impactos positivos sobre la identidad local en mayor medida que los eventos parcialmente turísticos (T2) (véase la Tabla 3). En este sentido apunta el hecho que el $81,8 \%$ de los encuestados está de acuerdo o muy de acuerdo con la opinión que los acontecimientos contienen y muestran los valores de la identidad local (en comparación con un 60,9\% en el caso de los eventos parcialmente turísticos). El $72,7 \%$ cree que el evento reproduce de algún modo las formas de vida local (frente al $54,5 \%$ en el caso de los eventos parcialmente turísticos), mientras que el 71,4\% piensan que los eventos orientados al turismo ofrecen una interpretación que permite avanzar en el conocimiento de la historia local (por un 54,9\% en el caso de los eventos parcialmente turísticos). Los eventos del tipo T2, sin embargo, se considera que tienen valores más altos que los de tipo $\mathrm{T} 1$ en algunos aspectos del contenido y programación del evento. Concretamente es así para las siguientes variables: presencia de artistas locales, tener un contenido cultural inalterado y presentar una mayor exactitud y rigor histórico en su programa. Estos tres elementos están relacionados con el grado de autenticidad percibida en un evento en términos de exactitud histórica y cultural. Sin embargo, los valores de la prueba de ji-cuadrado son bajos, lo que impide establecer asociaciones estadísticamente significativas para buena parte de las variables estudiadas.

Los eventos parcialmente turísticos (T2) muestran relaciones positivas con la mayoría de las variables que indican impacto sobre el fortalecimiento de la identidad local, especialmente con el hecho de mostrar el carácter de la identidad local y catalana (el 85,1\% de los encuestados está de acuerdo o muy de acuerdo con este aspecto), con ser una expresión de la forma de vida local $(75,1 \%)$ y con ofrecer una interpretación y fomentar el conocimiento de la historia local para los residentes $(70,8 \%)$. Los resultados de la prueba de ji-cuadrado confirman la existencia de una asociación estadísticamente significativa entre los eventos orientados al turismo y su capacidad para representar la cultura y la identidad local y catalana.

Los eventos con capacidad de atracción turística (T3) también reflejan valores de la identidad del lugar más altos en comparación con los otros tipos de eventos (T4 y T5). Las valoraciones que reúnen mayor grado de conformidad tienen que ver con la capacidad de los eventos para mostrar los elementos y valores de la identidad local (el 87,2\% de los encuestados está de acuerdo o muy de acuerdo en esta cuestión), de mostrar los valores de la identidad catalana $(80,2 \%)$, de ser una muestra representativa de la cultura local $(76,7 \%)$ y de ser una celebración que contribuye a fortalecer el idioma catalán (76,7 \%). Los resulta- 


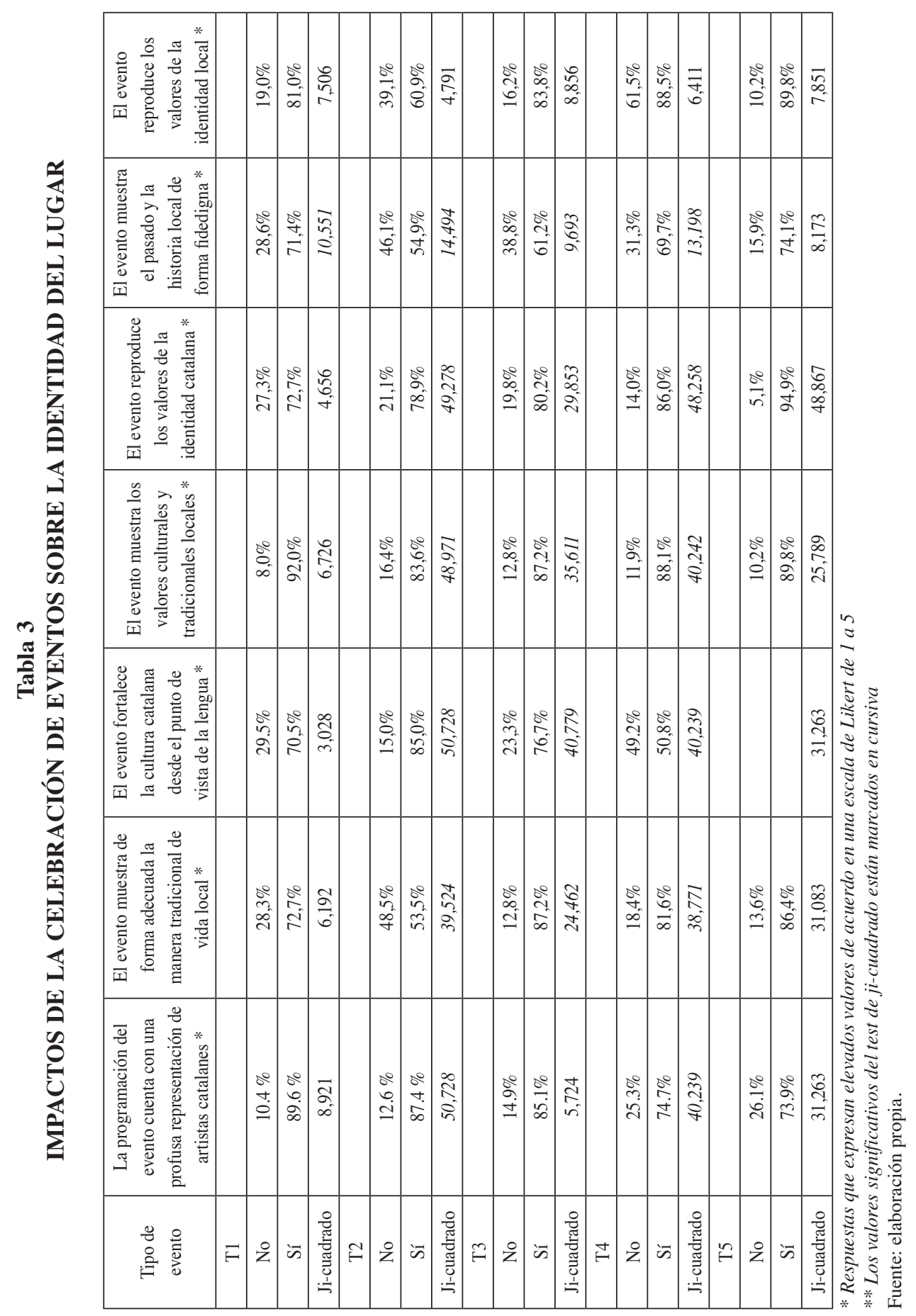


dos de la prueba de ji-cuadrado confirman la existencia de una asociación estadísticamente significativa entre los eventos con capacidad para atraer a turistas internacionales y los impactos positivos de los eventos sobre la identidad local. Por último, también existe una relación significativa entre los eventos transformados por el turismo (T4) y los eventos que diseñan su programación para turistas (T5) con los impactos positivos sobre la identidad local y la autenticidad percibida de la celebración. De hecho, estos dos tipos de eventos obtienen un alto porcentaje en las respuestas "de acuerdo" y "muy de acuerdo" en relación a los impactos sobre la identidad local $(88,5 \%$ y $89,8 \%$, respectivamente) y la identidad catalana $(86 \%$ y $94,9 \%)$. Igualmente tienen elevados porcentajes de respuestas que manifiestan su acuerdo con el hecho que los eventos de estos tipos funcionan como manifestaciones de la forma de vida local $(81,6 \%$ y $86,4 \%$ respectivamente) y que son instrumentos de interpretación de la historia local para los residentes $(69,7 \%$ y $74,1 \%$ respectivamente).

\section{Tabla 4}

\section{IMPACTOS DE LA CELEBRACIÓN DE EVENTOS SOBRE EL SENTIDO DEL LUGAR}

\begin{tabular}{|c|c|c|c|}
\hline Tipo de evento & $\begin{array}{l}\text { El evento ha incrementado } \\
\text { el número de medios de } \\
\text { comunicación internacionales } \\
\text { interesados por el evento }\end{array}$ & $\begin{array}{l}\text { El evento refuerza } \\
\text { la imagen turística } \\
\text { regional } *\end{array}$ & $\begin{array}{l}\text { El evento es fuente } \\
\text { de orgullo para los } \\
\text { residentes * }\end{array}$ \\
\hline \multicolumn{4}{|l|}{$\mathrm{T} 1$} \\
\hline No & $95,5 \%$ & $40,9 \%$ & $22,7 \%$ \\
\hline Sí & $4,5 \%$ & $59,1 \%$ & $77,3 \%$ \\
\hline Ji-cuadrado & 2,492 & 4,392 & 3,011 \\
\hline \multicolumn{4}{|l|}{$\mathrm{T} 2$} \\
\hline No & $98,7 \%$ & $31,4 \%$ & $12,7 \%$ \\
\hline Sí & $1,3 \%$ & $68,6 \%$ & $87,3 \%$ \\
\hline Ji-cuadrado & 9,918 & 52,664 & 35,661 \\
\hline \multicolumn{4}{|l|}{ T3 } \\
\hline No & $83,5 \%$ & $25,6 \%$ & $5,9 \%$ \\
\hline Sí & $16,5 \%$ & $74,4 \%$ & $94,1 \%$ \\
\hline Ji-cuadrado & 27,909 & 49,269 & 35,937 \\
\hline \multicolumn{4}{|l|}{$\mathrm{T} 4$} \\
\hline No & $79,2 \%$ & $26,8 \%$ & $11,5 \%$ \\
\hline Sí & $10,8 \%$ & $73,2 \%$ & $88,5 \%$ \\
\hline Ji-cuadrado & 11,577 & 45,983 & 23,886 \\
\hline \multicolumn{4}{|l|}{$\mathrm{T} 5$} \\
\hline No & $77,9 \%$ & $17,3 \%$ & $8,5 \%$ \\
\hline Sí & $12,1 \%$ & $82,7 \%$ & $91,5 \%$ \\
\hline Ji-cuadrado & 7,583 & 44,166 & 17,871 \\
\hline
\end{tabular}

* Respuestas que expresan elevados valores de acuerdo en una escala de Likert de 1 a 5

** Los valores significativos del test de ji-cuadrado están marcados en cursiva

Fuente: elaboración propia. 
Los impactos relacionados con la creación de un sentido del lugar se relacionan de forma significativa con el grado de turistificación de un evento. La celebración de los eventos mejora la imagen del lugar donde se celebran y hace emerger el sentido de orgullo entre la comunidad local (véase la Tabla 4). El impacto de los eventos turísticos (T5) sobre la mejora de la imagen del destino es especialmente elevado según las respuestas de los organizadores (el 82,7\% están de acuerdo o muy de acuerdo en este punto). Por su parte, los eventos con capacidad de atracción turística (T3) tienen el mayor porcentaje de respuestas conformes con el hecho que los eventos contribuyen a fomentar el sentido de orgullo entre la comunidad local. Por otro lado, en el caso de los eventos de orientación turística (T1) y los eventos parcialmente orientados al turismo (T2), la percepción del impacto de la celebración del evento sobre los medios de comunicación internacionales obtiene respuestas con bajos porcentajes de acuerdo (sólo el 4,5\% y 1,3\% está de acuerdo o muy de acuerdo, respectivamente). En contraste con esto, los eventos de atracción turística (T3) obtienen un alto porcentaje de respuestas de acuerdo con este punto (16,5\%). Nuevamente la mayor parte de las pruebas de ji-cuadrado muestran valores estadísticamente significativos, por lo que se puede establecer una relación entre el nivel de turistificación de un evento y los impactos percibidos sobre la mejora de la imagen y del sentimiento de orgullo local.

Los impactos de la celebración de eventos sobre el sentido de comunidad obtiene resultados algo diferentes al resto (véase la Tabla 5). Los eventos orientados al turismo (T1) tienen un menor impacto percibido sobre la participación de los residentes e instituciones y grupos locales en el evento (entidades cívicas y asociaciones culturales y sociales) que los eventos parcialmente orientados al turismo (T2). Sin embargo, la falta de significación en la mayoría de las pruebas de ji-cuadrado realizadas no permite hacer asociaciones sólidas al respecto. Los eventos con atracción turística (T3) obtienen el mayor porcentaje de respuestas acerca de la presencia de residentes entre los asistentes al evento (un 83,1\% de las respuestas manifiesta estar "de acuerdo" o "muy de acuerdo" en esta cuestión). La participación de entidades cívicas y asociaciones locales en la organización de eventos obtiene respuestas especialmente elevadas entre los eventos turísticos (T5) y los eventos con capacidad de atracción turística (T3) (91,5\% y 90,6\% respectivamente). La capacidad de movilización de voluntarios por parte de los eventos es muy importante entre los eventos del tipo T2 y T3. Así, la mayoría de las respuestas de estos dos tipos de eventos están "de acuerdo" o "muy de acuerdo" con este hecho ( $74.8 \%$ y 74,1\% respectivamente). En contraste, los eventos del tipo T4 (eventos transformados por el turismo) obtienen porcentajes más bajos $(62,5 \%)$ en cuanto a la importancia de los voluntarios en relación a los eventos de tipo T3 y T5 (74,15\% y 69,8\% respectivamente). La mayor parte de las pruebas de ji-cuadrado aportan resultados estadísticamente significativos y por lo tanto puede afirmarse que existe una asociación entre los eventos con capacidad de atracción turística (T3) y los eventos adaptados al turismo (T4) o turísticos (T5) y los impactos positivos percibidos sobre los elementos de capital social que fortalecen el sentido de comunidad entre los residentes locales.

El impacto positivo sobre el bienestar de una comunidad local también se relaciona con el componente turístico de los eventos (véase la tabla 6). Los eventos turísticos (T5) muestran los mayores porcentajes de impacto positivo en relación a la socialización de los residentes (un 94,7\% de respuestas están "de acuerdo" o "muy de acuerdo" en este punto) 
y de la capacidad de los eventos para renovar espacios públicos $(67,8 \%)$. Los eventos adaptados a los turistas (T4) son los que tienen el mayor porcentaje de respuestas positivas con respecto a la capacidad del evento de generar propuestas alternativas de ocio y de entretenimiento para la población local (el 95,3\% de las respuestas están "de acuerdo" o "muy de acuerdo" en este punto). Por su parte, los eventos con orientación turística (T1) son percibidos como los que más contribuyen a generar beneficios sociales a la comunidad $(100 \%)$ y a generar nuevas actividades de ocio y entretenimiento (76,2\%). La mayor parte de las pruebas de ji-cuadrado muestran la existencia de una relación estadísticamente significativa entre el nivel de turistificación de un evento y la presencia de impactos sobre el aumento del bienestar de la comunidad local.

Tabla 5

\section{IMPACTOS DE LA CELEBRACIÓN DE EVENTOS SOBRE EL SENTIDO DE COMUNIDAD}

\begin{tabular}{|c|c|c|c|c|}
\hline $\begin{array}{l}\text { Tipo de } \\
\text { evento }\end{array}$ & $\begin{array}{c}\text { La participación } \\
\text { de los residentes } \\
\text { en el evento es } \\
\text { considerable } *\end{array}$ & $\begin{array}{l}\text { Los residentes } \\
\text { forman parte } \\
\text { de la gestión y } \\
\text { organización del } \\
\text { evento * }\end{array}$ & $\begin{array}{c}\text { Las asociaciones y } \\
\text { entidades locales } \\
\text { participan en el } \\
\text { evento * }\end{array}$ & $\begin{array}{c}\text { La presencia de } \\
\text { voluntarios es } \\
\text { esencial para la } \\
\text { celebración del } \\
\text { evento } *\end{array}$ \\
\hline \multicolumn{5}{|l|}{$\mathrm{T} 1$} \\
\hline No & $17,3 \%$ & $35,0 \%$ & $33,4 \%$ & $30,0 \%$ \\
\hline Sí & $72,7 \%$ & $65,0 \%$ & $66,6 \%$ & $70,0 \%$ \\
\hline Ji-cuadrado & 1,187 & 1,024 & 7,595 & 2,441 \\
\hline \multicolumn{5}{|l|}{$\mathrm{T} 2$} \\
\hline No & $14,4 \%$ & $16,7 \% \%$ & $45,0 \%$ & $25,2 \%$ \\
\hline Sí & $75,6 \%$ & $83,3 \%$ & $55,0 \%$ & $74,8 \%$ \\
\hline Ji-cuadrado & 10,087 & 4,449 & 10,105 & 3,327 \\
\hline \multicolumn{5}{|l|}{$\mathrm{T} 3$} \\
\hline No & $16,9 \%$ & $9,4 \%$ & $18,1 \%$ & $25,9 \%$ \\
\hline Sí & $83,1 \%$ & $90,6 \%$ & $81,9 \%$ & $74,1 \%$ \\
\hline Ji-cuadrado & 14,188 & 16,256 & 16,387 & 16,119 \\
\hline \multicolumn{5}{|l|}{$\mathrm{T} 4$} \\
\hline $\mathrm{No}$ & $21,8 \%$ & $10,4 \%$ & $21,3 \%$ & $37,5 \%$ \\
\hline Sí & $79,2 \%$ & $89,6 \%$ & $78,7 \%$ & $62,5 \%$ \\
\hline Ji-cuadrado & 14,888 & 11,407 & 10,396 & 4,695 \\
\hline \multicolumn{5}{|l|}{ T5 } \\
\hline No & $13,2 \%$ & $8,5 \%$ & $30,2 \%$ & $30,2 \%$ \\
\hline Sí & $76,8 \%$ & $91,5 \%$ & $69,8 \%$ & $69,8 \%$ \\
\hline Ji-cuadrado & 6,001 & 8,299 & 2,796 & 2,109 \\
\hline
\end{tabular}

* Respuestas que expresan elevados valores de acuerdo en una escala de Likert de 1 a 5

** Los valores significativos del test de ji-cuadrado están marcados en cursiva

Fuente: elaboración propia. 


\section{Tabla 6 \\ IMPACTOS DE LA CELEBRACIÓN DE EVENTOS SOBRE LA SENSACIÓN DE BIENESTAR DE LA COMUNIDAD LOCAL}

\begin{tabular}{|c|c|c|c|c|c|}
\hline $\begin{array}{l}\text { Tipo de } \\
\text { evento }\end{array}$ & $\begin{array}{c}\text { El evento } \\
\text { favorece la } \\
\text { socialización } \\
\text { de los } \\
\text { residentes * }\end{array}$ & $\begin{array}{c}\text { El evento permite } \\
\text { aumentar las } \\
\text { posibilidades de ocio } \\
\text { y de entretenimiento } \\
\text { entre la población } \\
\text { local } * \\
\end{array}$ & \begin{tabular}{|c} 
El evento \\
ofrece nuevas \\
actividades \\
culturales para \\
la población \\
local $*$ \\
\end{tabular} & $\begin{array}{l}\text { El evento } \\
\text { resulta } \\
\text { beneficioso } \\
\text { para la } \\
\text { población } \\
\text { local * } \\
\end{array}$ & $\begin{array}{l}\text { El evento } \\
\text { permite la } \\
\text { mejora de } \\
\text { los espacios } \\
\text { públicos } \\
\text { urbanos * } \\
\end{array}$ \\
\hline \multicolumn{6}{|l|}{$\mathrm{T} 1$} \\
\hline No & $9,1 \%$ & $4,8 \%$ & $23,8 \%$ & $0,0 \%$ & $42,1 \%$ \\
\hline Sí & $80,9 \%$ & $95,2 \%$ & $76,2 \%$ & $100,0 \%$ & $57,9 \%$ \\
\hline Ji-cuadrado & 2,927 & 3,956 & 7,129 & 7,671 & 5,043 \\
\hline \multicolumn{6}{|l|}{$\mathrm{T} 2$} \\
\hline No & $11,4 \%$ & $9,3 \%$ & $39,9 \%$ & $12,8 \%$ & $39,2 \%$ \\
\hline Sí & $88,4 \%$ & $90,7 \%$ & $60,1 \%$ & $87,2 \%$ & $60,8 \%$ \\
\hline Ji-cuadrado & 32,654 & 5,912 & 10,938 & 8,235 & 37,121 \\
\hline \multicolumn{6}{|l|}{$\mathrm{T} 3$} \\
\hline No & $7,1 \%$ & $7,1 \%$ & $44,1 \%$ & $9,4 \%$ & $34,2 \%$ \\
\hline Sí & $92,9 \%$ & $92,9 \%$ & $55,9 \%$ & $90,6 \%$ & $65,8 \%$ \\
\hline Ji-cuadrado & 27,763 & 10,812 & 4,966 & 14,847 & 36,589 \\
\hline \multicolumn{6}{|l|}{$\mathrm{T} 4$} \\
\hline No & $7,1 \%$ & $4,7 \%$ & $29,1 \%$ & $12,8 \%$ & $32,6 \%$ \\
\hline Sí & $92,9 \%$ & $95,3 \%$ & $70,9 \%$ & $87,2 \%$ & $67,4 \%$ \\
\hline Ji-cuadrado & 27,664 & 7,594 & 26,287 & 4,233 & 32,018 \\
\hline \multicolumn{6}{|l|}{ T5 } \\
\hline No & $5,3 \%$ & $5,2 \%$ & $32,2 \%$ & $13,6 \%$ & $32,2 \%$ \\
\hline Sí & $94,7 \%$ & $94,8 \%$ & $67,8 \%$ & $86,4 \%$ & $67,8 \%$ \\
\hline Ji-cuadrado & 19,099 & 4,071 & 13,963 & 4,967 & 20,548 \\
\hline
\end{tabular}

* Respuestas que expresan elevados valores de acuerdo en una escala de Likert de 1 a 5

** Los valores significativos del test de ji-cuadrado están marcados en cursiva

Fuente: elaboración propia.

Los impactos negativos derivados de la celebración de eventos también están relacionados con el nivel de turistificación (véase la tabla 7). Los eventos con capacidad de atracción turística (T3) obtienen los porcentajes de respuesta más elevados sobre el impacto negativo que causa la presencia de turistas en un evento (el 11,6\% de las respuestas están de acuerdo o muy de acuerdo en este punto), aunque el porcentaje es menor en los eventos transformados por el turismo (T4) (7\%). La percepción de los impactos debido a la existencia de tensiones con la administración local derivada de la celebración de eventos es más elevada entre los eventos orientados al turismo (T1) y en los turísticos (T5). Sin embargo, los bajos resultados en las pruebas de significación no permiten establecer inferencias entre estas dos variables. En cambio, los eventos con capacidad de atracción turística (T3) sí que guardan una relación 
estadísticamente significativa con los impactos sociales negativos. Concretamente un 6,9\% de las respuestas están "de acuerdo" o "muy de acuerdo" con que la celebración del evento causa tensión con los residentes. Finalmente, existen dos tipos de eventos que mayoritariamente consideran que los fondos públicos destinados a la celebración de eventos deberían ser dirigidos a otras finalidades sociales y de utilidad para la comunidad. Se trata de los eventos transformados por el turismo (T4) (el 73,2\% de respuestas están "de acuerdo" o "muy de acuerdo" en este punto) y los eventos con capacidad de atracción turística (T3) (69,7\%). Las pruebas de ji-cuadrado confirman la existencia de una relación estadísticamente significativa entre el nivel turistificación de un evento y la mayoría de las variables analizadas sobre su impacto social negativo generado percibido por los organizadores.

\section{Tabla 7 \\ IMPACTOS SOCIALES Y CULTURALES NEGATIVOS DE LA CELEBRACIÓN DE EVENTOS}

\begin{tabular}{|c|c|c|c|c|}
\hline $\begin{array}{l}\text { Tipo de } \\
\text { evento }\end{array}$ & $\begin{array}{c}\text { La presencia de } \\
\text { turistas asistentes al } \\
\text { evento es percibida } \\
\text { como una molestia } \\
\text { por parte de los } \\
\text { residentes locales } *\end{array}$ & $\begin{array}{c}\text { La organización } \\
\text { y celebración del } \\
\text { evento provoca } \\
\text { tensiones con la } \\
\text { administración } \\
\text { local } *\end{array}$ & $\begin{array}{l}\text { La presencia de } \\
\text { turistas asistentes al } \\
\text { evento es percibida } \\
\text { como una molestia } \\
\text { por parte de los } \\
\text { residentes locales } *\end{array}$ & $\begin{array}{l}\text { Los recursos destinados } \\
\text { a la financiación } \\
\text { del evento deberían } \\
\text { dedicarse a cubrir } \\
\text { necesidades más } \\
\text { urgentes de la localidad* }\end{array}$ \\
\hline \multicolumn{5}{|l|}{$\mathrm{T} 1$} \\
\hline No & $90,5 \%$ & $90,5 \%$ & $100,0 \%$ & $33,4 \%$ \\
\hline Sí & $9,5 \%$ & $9,5 \%$ & $0,0 \%$ & $66,6 \%$ \\
\hline Ji-cuadrado & 3,868 & 4,241 & 3,119 & 2,626 \\
\hline \multicolumn{5}{|l|}{$\mathrm{T} 2$} \\
\hline No & $90,1 \%$ & $95,8 \%$ & $96,6 \%$ & $28,4 \%$ \\
\hline Sí & $9,9 \%$ & $4,2 \%$ & $3,4 \%$ & $71,6 \%$ \\
\hline Ji-cuadrado & 22,938 & 2,951 & 0,061 & 17,131 \\
\hline \multicolumn{5}{|l|}{ T3 } \\
\hline No & $88,4 \%$ & $95,4 \%$ & $93,1 \%$ & $30,3 \%$ \\
\hline Sí & $11,6 \%$ & $4,6 \%$ & $6,9 \%$ & $69,7 \%$ \\
\hline Ji-cuadrado & 16,807 & 18,427 & 25,177 & 13,076 \\
\hline \multicolumn{5}{|l|}{$\mathrm{T} 4$} \\
\hline $\mathrm{No}$ & $93,0 \%$ & $96,5 \%$ & $96,5 \%$ & $26,8 \%$ \\
\hline Sí & $7,0 \%$ & $3,5 \%$ & $3,5 \%$ & $73,2 \%$ \\
\hline Ji-cuadrado & 18,366 & 5,579 & 1,683 & 12,271 \\
\hline \multicolumn{5}{|l|}{ T5 } \\
\hline No & $91,4 \%$ & $94,9 \%$ & $93,2 \%$ & $30,5 \%$ \\
\hline Sí & $8,6 \%$ & $5,1 \%$ & $6,8 \%$ & $69,5 \%$ \\
\hline Ji-cuadrado & 8,674 & 3,066 & 4,521 & 7,002 \\
\hline
\end{tabular}

* Respuestas que expresan elevados valores de acuerdo en una escala de Likert de 1 a 5

** Los valores significativos del test de ji-cuadrado están marcados en cursiva

Fuente: elaboración propia. 


\section{CONCLUSIONES}

La primera conclusión es que los impactos sociales y culturales están relacionados con el nivel de turistificación del evento. Los eventos que incluyen el turismo entre sus objetivos obtienen valores de impacto social y cultural (tanto positivos como negativos) por encima del nivel de los que no incluyen el turismo como objetivo principal o no se adaptan a los gustos de los turistas. En este sentido, la turistificación de los eventos acaba teniendo como resultado la generación de efectos positivos sobre el sentido del lugar, el fortalecimiento del sentimiento de comunidad, la identidad local y sobre el bienestar de la comunidad local. El nivel de implicación turística de un evento también guarda relación con el aumento de la percepción de los impactos sociales negativos derivados de su celebración. Estos aspectos se observan con mayor claridad si consideramos por separado los cinco tipos de eventos analizados en el estudio.

Los eventos orientados al turismo (T1), ejercen efectos positivos sobre la identidad del lugar donde se celebran. La mayoría de los eventos y festivales catalanes se basan en narrativas culturales o históricas de carácter local y se fundamentan en los valores del patrimonio local. Esto es lógico teniendo en cuenta que dichos eventos tienen frecuentemente la intención de singularizar y diferenciar la oferta turística local a partir del patrimonio intangible. Resulta muy significativo el hecho que los eventos sean percibidos más claramente como instrumentos para el refuerzo de la identidad local que de la identidad catalana. Sin embargo, la percepción de los valores de autenticidad de la programación de los eventos (interpretación histórica, manifestación de valores culturales del pasado no alterados), es menor entre estos eventos con carácter turístico, más cercanos a los espectáculos o a las propuestas lúdicas que a una celebración popular o tradicional local.

Los eventos parcialmente orientados al turismo (T2) también generan impactos sociales y culturales positivos. La mayoría de estos eventos no fueron inicialmente concebidos para complementar la oferta turística local aunque en la actualidad tienen un marcado uso turístico. En este tipo de eventos los impactos sobre la identidad local y sobre el sentido de comunidad son percibidos de forma aún más marcada que los eventos del tipo anterior (T1) puesto que el valor simbólico o tradicional del evento queda muy por encima de la función comercial o turística del mismo, aun y cuando pueden llegar a contar con numerosos turistas entre los asistentes.

Los eventos con capacidad de atracción de turistas (T3) muestran un comportamiento muy diferente. Son eventos de reconocido prestigio, con potencial para atraer visitantes internacionales y que a menudo han evolucionado a partir de eventos comunitarios de pequeña dimensión para acabar convirtiéndose en eventos de alcance nacional o internacional. De entre los eventos que pertenecen a este tipo, aquellos que están enraizados en la comunidad (especialmente los que están arraigados a la historia local o reflejan la identidad local), los que cuentan con una elevada participación local y los que permiten a los visitantes interactuar con la comunidad local ofrecen una buena oportunidad para los turistas que buscan experiencias basadas en la autenticidad y la socialización. A medida que el turismo va permeabilizando este tipo de eventos aparecen efectos negativos sobre la población local. La percepción de los impactos es particularmente elevada en relación a las molestias e interrupciones de la vida cotidiana o por el ruido generado y sobre todo 
por las molestias debido a la presencia de turistas durante la celebración del evento. Es hasta cierto punto sorprendente ver, sin embargo, que también presentan efectos sociales y culturales positivos. Este tipo de eventos es el único en que la representación de los valores de la identidad catalana es percibida de forma más intensa que la representación de los valores de la identidad local. Esto probablemente tenga que ver con la inclusión en este tipo de eventos de referencias simbólicas, conceptos y narrativas con los que están más familiarizados los turistas asistentes.

Por último, los eventos transformados por el turismo (T4) y los eventos turísticos (T5) generan impactos sociales y culturales positivos sobre la comunidad local. Ambos tipos de eventos contienen elementos de socialización y de entretenimiento y, frecuentemente, complementan las actividades de ocio y culturales para la comunidad local. Sin embargo, los impactos negativos asociados a estos tipos de eventos son también más altos en comparación con el resto, sobre todo en relación al excesivo coste económico y a las molestias y malestar percibido por los residentes durante su celebración. De hecho, los eventos de tipo T4 y T5 acostumbran a estar diseñados como complemento turístico (festivales de verano, por ejemplo) o se apoyan en festivales y fiestas preexistentes (las Fiestas Mayores, por ejemplo) para mejorar la oferta de ocio y turística en temporada alta. Por lo tanto, resulta lógico que su celebración influya tanto sobre la percepción de mejora del bienestar y la calidad de vida de los residentes locales, como en las molestias ocasionadas a los residentes (especialmente a aquellos que no se encuentran de vacaciones).

Este estudio proporciona suficientes evidencias empíricas para abrir un debate sobre el papel del impacto social y cultural de los eventos organizados en los destinos turísticos. A pesar de que algunos estudios señalan que el éxito de los eventos y festivales implica convertirse en objeto de mercantilización (Getz \& Frisby, 1988; Chacko \& Shaffer, 1993), el estudio de los eventos catalanes demuestra que, si bien existen impactos culturales y sociales negativos, el carácter turístico de los eventos no impide la aparición de impactos socioculturales positivos. Los eventos con carácter turístico generan efectos positivos sobre diferentes variables que indican un fortalecimiento del sentido del lugar, el sentido de la identidad, el sentido de comunidad y el aumento del bienestar de la comunidad local.

Es especialmente interesante destacar que el grado de turistificación de un evento es un elemento que contribuye, de forma decisiva, a la creación de un fuerte sentido del lugar entre los miembros de la comunidad local. Esto está relacionado tanto con factores externos, tales como la mejora de la imagen del destino, como internos a la comunidad local (un aumento del sentimiento de orgullo local debido a la celebración del evento).

Según lo sugerido por algunos autores existe una relación entre la presencia de elementos del capital social y la organización de eventos (Misener, 2013). En el caso de los eventos catalanes se confirma que el capital social es un elemento que refuerza el sentido local de la comunidad y crea las condiciones para que las relaciones basadas en la participación y la confianza se conviertan en recursos potenciales que se pueden ser utilizadas para organizar estrategias de cohesión social. Los eventos catalanes se caracterizan por una elevada participación de las asociaciones locales en la organización de los mismos y por unos altos niveles de participación de los residentes. Además permiten 
la formación de redes de relaciones sociales espontáneas, fomentan la presencia del voluntariado y aumentan la atracción de visitantes. Los eventos actúan como estructuras capaces de movilizar a diferentes grupos e instituciones pertenecientes a una comunidad, lo que demuestra que pueden generar capital social.

El capital social que se encuentra en los eventos de Cataluña se nutre de tres elementos diferentes. En primer lugar, los acontecimientos actúan como espacios de socialización entre los miembros de la comunidad local a través de la participación. En muchos casos (como en las fiestas locales, la tradicional "Patum" de Berga o las Pasiones durante la Semana Santa) la celebración ejerce de punto de referencia social para toda la comunidad local. Por otra parte, la participación colectiva a través de un evento refuerza el sentido de pertenencia al grupo y la localidad. Esta situación se ve aumentada cuando el evento cuenta con voluntarios y redes de colaboradores, pero también presenta algunos riesgos si se producen patrones de exclusión social en las instituciones o asociaciones locales. En definitiva, la creación de capital social derivado de la celebración de eventos tiene un gran potencial para generar sinergias que convergen en la mejora de la confianza y la participación entre los diferentes grupos de interés, aspectos que, finalmente, favorecen el diseño de estrategias colectivas para la obtención de fines comunes.

Hasta aquí hay dos aspectos que merece la pena destacar. En primer lugar, hemos demostrado que la organización de eventos con carácter turístico fortalece la identidad local, la cohesión social y el bienestar de la comunidad local en los destinos turísticos catalanes. Este resultado permite introducir matices a las interpretaciones acerca de que los eventos turísticos promueven el choque cultural o trivializan la cultura y las tradiciones locales.

En segundo lugar, los resultados apuntan a que la construcción social de la identidad de los destinos turísticos parece explicarse también por la celebración de eventos. Sin duda los impactos percibidos por parte de los organizadores se ven afectados por su vinculación a un lugar donde el turismo es central en la producción y donde modela el modo de vida local. Los eventos con carácter turístico (como algunos festivales, jornadas gastronómicas o mercados para la promoción de productos locales) pueden ser percibidos como activos de valor turístico para el destino por parte de los organizadores y, por este motivo, existe una elevada percepción positiva del impacto que los eventos ejercen sobre la mejora de la imagen de los destinos, sobre el interés que los medios de comunicación internacionales ponen en el destino o sobre el sentimiento de orgullo que los eventos causan en la comunidad local. Por otra parte, el bajo impacto social y cultural percibido sobre la comunidad local sugiere que se interpretan más como efectos colaterales, que como el resultado de un choque cultural. La autenticidad percibida de los eventos (por ejemplo en relación al rigor histórico o a su capacidad como instrumento para interpretar el pasado) no es muy elevada, pero en cambio sí existe una percepción general que considera que los eventos favorecen el sentido de la identidad local y se muestran como una expresión de la cultura y de la tradición local. Esta aparente paradoja se explica por el hecho de que el turismo se considera un elemento integrado en la forma de vida local.

En vista de todo lo expuesto se destaca, a continuación, dos implicaciones prácticas de interés para los responsables de la gestión de eventos en los destinos turísticos. En primer lugar, la celebración de eventos puede resultar un incentivo para el desarrollo de 
nuevos productos turísticos locales basados en narrativas relacionadas con la identidad local y el patrimonio cultural. Aunque el aumento de la oferta de actividades turísticas o su tematización excesiva con el fin de satisfacer determinados mercados puede poner en riesgo autenticidad de algunos eventos, seguirán siendo elementos de fortalecimiento de la identidad en la medida que no excluyan a la comunidad local. Los resultados obtenidos en el análisis de los eventos catalanes celebrados en destinos turísticos muestran que una estrategia de diversificación turística basada en la organización de eventos no sólo podría mejorar la imagen de un destino y su posición en el mapa turístico internacional, sino que también es compatible con el refuerzo del sentido del lugar y del sentimiento de orgullo local. Por otro lado, los organizadores de eventos perciben sinergias entre el evento y la mejora de la imagen como destino turístico. Por ejemplo, algunos eventos como «Tarraco Viva» en Tarragona (un evento de recreación histórica centrada en la época romana), los eventos se utilizan de forma proactiva en la promoción turística de la ciudad y se vinculan a la imagen del destino.

Por último, la aparición de impactos negativos moderados derivados de la celebración de eventos en la comunidad local resulta también evidente. No obstante esta aparente debilidad puede ser reconducida hacia una oportunidad si dichos impactos son utilizados como un argumento en favor del fortalecimiento de la cohesión social mediante el diseño adecuado de estrategias de multiplicación de los impactos sociales. En este sentido es importante saber reconocer la oportunidad del legado que la planificación de eventos puede generar a largo plazo no solamente a nivel económico, sino también como herramienta de integración social, la generación de capital social, y el fortalecimiento del sentido de pertenencia a la comunidad. Esto puede ser conseguido en aquellas comunidades turísticas que confían en mostrar su identidad y tradiciones a través de los eventos que acogen. Una pregunta de investigación futura es analizar, en un contexto de incesante proceso de transformación cultural, hasta qué punto los eventos turísticos actuales incorporarán la tradición y cultura local en el imaginario colectivo y hasta qué punto las narrativas de este tipo de eventos los convertirán en los futuros eventos tradicionales de los destinos donde tienen lugar actualmente.

\section{BIBLIOGRAFÍA}

ARCODIA, Ch. \& WHITFORD, M. (2006): "Festival Attendance and the Development of Social Capital", Journal of Convention \& Event Tourism, no 8(2), pp. 1-18.

AVERY, P.M. (2000): "City cultures as the object of cultural tourism 2000" en Robinson, M. (ed.) Developments in urban and rural tourism. Sunderland, Business Education Publishing, pp. 1-35.

BOURDIEU, P. (1983): “Economic capital, cultural capital, social capital”. Soziale-Welt, Supplement 2, pp. 183-198.

CARLSEN, J.; ROBERTSON, M.; ALI-KNIGHT, J. (2008): "Festivals and events: beyond economic impacts", Event Management, $\mathrm{n}^{\circ}$ 11(1-2), pp. 1-2.

CHACKO, H. \& SCHAFFER, J. (1993): “The evolution of a festival: Creole Christmas in New Orleans", Tourism Management, $\mathrm{n}^{\circ} 14(4)$, pp. 475-482. 
COLEMAN, J.S. (1988): "Social Capital and the Creation of Human Capital", American Journal of Sociology, n 94, pp. 95-121.

COOKE, P., \& WILLS, D. (1999): "Small firms, social capital and the enhancement of business performance through innovation programmes", Small Business Economics, $\mathrm{n}^{\mathrm{o}} 13(3), \mathrm{pp} .219-234$.

DE BRES, K \& DAVIS, J. (2001): "Celebrating group and place identity: A case study of a new regional festival”, Tourism Geogaphies, no 3(3), pp. 326-337.

DEERY, M \& JAGO, L. (2010): "Social impacts of events and the role of anti-social behaviour", International Journal of Event and Fetival Management, $\mathrm{n}^{\circ} 1(1)$, pp. 8-28.

DERRETT R. (2003): "Making sense of how festivals demonstrate a community's sense of place", Event Management, n ${ }^{\circ} 8(1)$, pp. 49-58.

FAULKNER B.; CHALIP L.; BROWN G.; JAGO L.; MARCH R.; WOODSIDE A. (2000): "Monitoring the tourism impacts of the Sydney 2000 Olympics", Event Management, $\mathrm{n}^{\circ} 6(4)$, pp. 231-246.

EISINGER, P. (2000): "The politics of bread and circuses. Building the city for the visitor class", Urban Affairs Review, no 35, pp. 316-333.

FREDLINE. E. \& FAULKNER, B. (2002): "Residents reactions to a major tourist event: the Gold Coast Indy car race", Festival Management and Event Tourism, n ${ }^{\circ}$ 5(4), pp. 185-205.

FREDLINE E.; JAGO L.; DEERY M. (2003): “The development of a generic scale to measure the social impacts of events", Event Management, n ${ }^{\circ}$ 8(1), pp. 23-37.

GETZ, D. (1989): "Special events: defining the product", Tourism Management, $\mathrm{n}^{\circ}$ 10(2), pp. 125-137.

GETZ, D. (1997): Event Management and Event tourism. Cognizant Communication, New York.

GETZ, D. (2007): Event Studies. Theory, research and policy for planned events. London, Elsevier.

GETZ, D. \& FRISBY, W. (1988): "Evaluating management effectiveness in communityrun festivals", Journal of Travel Research (summer), pp. 22-27.

GIAMPICCOLI, A.; SHAWN, S. \& NAURIGHT, J. (2013): "Destination South Africa: comparing global sports mega-events and recurring localised sports events in South Africa for tourism and economic development", Current Issues in Tourism.

GONZÁLEZ, F. \& MORALES, S. (2009): Ciudades efímeras transformando el turismo urbano a través de la producción de eventos, Barcelona: Editorial UOC.

GONZÁLEZ, F.; GOMIS, J.M.; MIRALBELL, O. \& VIU, M. (2012): "The role of identity in events scheduling", Annals of Tourism Research, no 39(3), pp. 1683-1690.

GONZÁLEZ, F., \& MIRALBELL, O. (2011): The role of social and intangible factors in cultural event planning in Catalonia, International Journal of Event and Festival Management, $\mathrm{n}^{\circ}$ 2(1), pp. 37-53.

GONZÁLEZ, F., \& MIRALBELL, O. (2009): Managing music festivals for tourism purposes in Catalonia (Spain), Tourism Review, $\mathrm{n}^{\circ}$ 64(4), pp. 53-65.

EKMAN, A.K. (1999): "The revival of cultural celebrations in regional Sweeden: Aspects of tradition and transition", Sociologia Ruralis, n 39(3), pp. 280-293. 
GUIU, C. (2008): "New Heritage Festivals around Catalonia and Spain: Places and Performances of the Regional Identity", Journal of Mediterranean Studies, $\mathrm{n}^{\circ} 18(1)$, pp. 93-118.

GUNSOY, E \& HANNAM, K (2013): "Fetivals, community development and sustainable tourism in the Karpaz region of Northern Cyprus", Journal of Policy Research in Tourism, Leisure \& Events, $\mathrm{n}^{\circ}$ 5(1), pp. 81-94.

HARPHAM, T., GRANT, E., \& THOMAS, E. (2002): "Measuring social capital within health surveys: key issues", Health policy and planning, $\mathrm{n}^{\circ} 17(1), \mathrm{pp} .106$.

HIGHAM J.E.S.; RITCHIE B. (2001): “The evolution of festivals and other events in rural southern new Zealand", Event Management, n 7(1), pp. 39-49.

JEONG, S. \& SANTOS, C.A. (2004): "Cultural politics and contested place identity", Annals of Tourism Research, $\mathrm{n}^{\mathrm{o}} 31(3), \mathrm{pp}$. 640-656.

JEPSON, A. \& CLARKE, A. (2013): "Events and community development" en Finkel, R. et al. (eds.) Research themes for events. Wallingford: CABI, pp. 6-17.

KEOGH, B. (1990): "Public participation in community tourism planning", Annals of Tourism Research, $\mathrm{n}^{\circ}$ 17(3), pp. 449-465.

KNORRINGA, P. \& VAN STAVEREN, I. (2007): "Beyond Social Capital: A critical approach", Review of Social Economy, n LXV(1), pp. 1-9.

MACKELLAR, J. (2006): “An integrated view of innovation emerging from a regional festival", International Journal of Event Management Research, $\mathrm{n}^{\circ} 2(1)$, pp. 37-48.

MCCARTNEY, G., \& OSTI, L. (2007): "From cultural events to sport events: a case study of cultural authenticity in the Dragon Boat Races", Journal of Sport \& Tourism, $\mathrm{n}^{\mathrm{o}} 12(1), \mathrm{pp} .25-40$.

MACLEOD, N. (2006): "The placeless festival: identity and place in the post-modern festival" en Picard, D. \& robinson, M. (eds.) Festivals, Tourism and Social Change. Remaking Worlds. Clevedon: Channel View, pp. 222-237.

MAIR, J \& WHITFORD, M. (2013): “An exploration of events research: event topics, themes and emerging trends", International Journal of Event and Festival Management, $\mathrm{n}^{\mathrm{o}} 4(1)$, pp. 6-30.

MEHMETOGLU, M. \& ELLINGSON, K.A. (2005): "Do small-scale festivals adopt "market orientation" as a management philosophy?", Event Management, n 9, pp. 119-132.

MISENER, L. (2013): “Events and social capital”·en Finkel, R. et al. (eds.) Research themes for events. Wallingford: CABI, pp. 18-30.

MISENER, L. \& MASON, D (2006a): “Creating community networks: Can sporting events offer meaningful sources of social capital?", Managing Leisure, $\mathrm{n}^{\mathrm{o}} 11$, pp. $39-56$.

MISENER, L. \& MASON, D (2006b): “Developing Local Citizenship through Sporting Events: Balancing Community Involvement and Tourism Development", Current Issues in Tourism, $\mathrm{n}^{\circ}$ 9(4\&5), pp. 384-398.

MORALES, S.; SANCHEZ, J. P; VIDAL, M. \& FLORES, M. (2009): Anàlisi estratègica de les fires i les festes de Maig o dels Enamorats. Vilafranca del Penedès. Ajuntament de Vilafranca del Penedès. 
MORALES, S. (2013): "La utilidad social de los eventos. Revisitando la relación entre eventos y el desarrollo a partir del estudio del capital social” en Gascón, J; Morales, $\mathrm{S}$ y Tresserras, J. (ed.) Cooperación en turismo. Nuevos desafios, nuevos debates. Barcelona. pp. 257-278.

MOSCARDO, G. (2008): "Analyzing the role of festivals and events in regional development”, Event Management, $\mathrm{n}^{\mathrm{o}}$ 11(1-2), pp. 23-32.

MULES, T. (2004): "Evolution in event management: The Gold Coast's Wintersun festival", Event Management, n ${ }^{\circ}$ 9, pp. 95-101.

NAHAPIET, J. \& GHOSHAL, S. (1998): "Social capital, intellectual capital, and the organizational advantage", Academy of Management Review, n ${ }^{\circ} 23(2)$, pp. 242-268.

OKAZAKI, T. (2008): “A Community based Tourism Model. Its Conception and Use", Journal of Sustainable Tourism, $\mathrm{n}^{\mathrm{o}}$ 16(5), pp. 511-529.

PIZAM, A. (1978): "Tourist impacts: the social costs to the destination community as perceived by the residents", Journal of Travel Research, $\mathrm{n}^{\circ}$ 16(4), pp. 8-12.

PIZAM, A.; MILMAN, A.; KING, B. (1994): "The perceptions of tourism employees and their families towards tourism", Tourism Management, $\mathrm{n}^{\circ} 15(1), \mathrm{pp} .53-61$.

PUGH,C. \& WOOD, E. (2004): "The strategic use of events within local government: a study of London borough councils", Event Management, $\mathrm{n}^{\circ}$ 9(1-2), pp. 61-71.

PUTNAM, R. D. (1993): “The prosperous community", The American Prospect, $\mathrm{n}^{\circ}$ 4(13), pp. 35-42.

PUTNAM, R. D. (1995): “Tuning in, tuning out: The strange disappearance of social capital in America", PS-WASHINGTON, n 28 , pp. 664-664.

PUTNAM, R.D. (1995): “Bowling alone: America's declining social capital”, Journal of Democracy, n 6(1), pp. 65-78.

PUTNAM, R. D. (2000): Bowling alone: the Collapse and Revival of American Community. New York: Simon and Schuster.

REID, S (2008): "Identifying social consequences of rural events", Event Management, $\mathrm{n}^{\circ} 11(1 / 2)$, pp. 89-98.

RICHARDS, G., \& PALMER, R. (2010): Eventful cities : management and urban revitalisation. Amsterdam: Elsevier.

RICHARDS, G \& WILSON, J. (2006): "Developing creativity in tourism experiences: a solution to the serial reproduction of culture?", Tourism Management, $\mathrm{n}^{\circ} 27(6)$, pp. 1209-1223.

RICHARDS, G. \& WILSON, J. (2004): "The impact of cultural eents on city image: Rotterdam, cultural capital of Europe 2001”, Urban Studies, no 41(10), pp. 1931-1951.

RITCHIE, B.W.; SANDERS, D.; MULES, T. (2007): "Televised events: shaping destination images and perceptions of capital cities from the couch", International Journal of Event Management Research, $\mathrm{n}^{\mathrm{o}}$ 3(2), pp. 12-23.

RONSTRÖM, O. (2011): "Festivalisation: what a festival says - and does. Reflecions over festivals and festivalisation". Presented at the International Colloquium "Sing as a simple song" on representation, exploitation, transmission and invention of cultures in the context of world music festivals. Neuchâtel, Switzerland.

ROTHMAN, R. (1978): "Residents and transients: community reactions to seasonal visitors", Journal of Travel Research, n ${ }^{\circ} 16(3)$, pp. 8-13. 
SHARPLEY, R. \& STONE, P.R. (2012): "Socio-cultural impacts of events" en The Routledge Handbook of Events. London: Routledge, pp. 347-361.

SHELDOM, P.J. \& VAR, T (1984): "Resident attitudes to tourism in North Wales", Tourism Management, $\mathrm{n}^{\circ}$ 5(1), pp. 40-47.

SHIBLI, S. \& COLEMAN, R. (2005): "Economic impact and place marketing evaluation: a case study of the world snooker championship", International Journal of Event Management Research, $\mathrm{n}^{\mathrm{o}} 1(1), \mathrm{pp} .13-29$.

SMALL, K. (2008): "Social dimensions of community festivals: an application of factor analysis in the development of the social impact perception (SIP) scale", Event Management, $\mathrm{n}^{\mathrm{o}}$ 11(1-2), pp. 45-55.

SMALL, K.; EDWARDS, D.; SHERIDAN, L. (2005): “A flexible framework for evaluating the socio-cultural impacts of a (small) festival", International Journal of Event Management Research, $\mathrm{n}^{\circ} 1(1)$, pp. 66-77.

SMITH,A. (2012): Events and urban regeneration. The strategic use of events to revitalise cities. London: Routledge.

STOKES, R. \& JAGO, L. (2007): “Australia's public sector environment for shaping event tourism strategy", International Journal of Event Management, $\mathrm{n}^{\circ}$ 3(1), pp. 42-53.

WATTERMAN, S. (1998): "Carnival for elites? The cultural politics of arts festivals", Progress in Human Geography, no 2(1), pp. 54-74.

WILKS, P. (2012): "Social capital in the music festival experience". The Routledge Handbook of Events. London. Routledge, pp. 260-272.

WINKLE, C.M. \& WOOSNAM, K.M. (2014): "Sense of community and perceptions of festival social impacts", International Journal of Event and Festival Management, $\mathrm{n}^{\mathrm{o}}$ 5(1), pp. 22-38.

WOOD, R. (1998): "Tourist ethnicity: a brief itinerary", Ethnic and Racial Studies, no 21(2), pp. 218-241.

WOOLCOCK, M. (1998): "Social capital and economic development: Toward a theoretical synthesis and policy framework", Theory and society, $\mathrm{n}^{\circ} 27(2), \mathrm{pp}$. 151-208.

WOOLCOCK, M., \& NARAYAN, D. (2000): "Social capital: Implications for development theory, research, and policy", The World Bank Research Observer, n 15(2), pp. 225-249.

XIAO, H \& SMITH, S.L.J. (2004): "Resident's perceptions of Kitchener-Waterloo Oktoberfest. An inductive analysis", Event Management, no 8(3), pp. 161-175. 